\title{
Scaling exponents of Forced Polymer Translocation through a nano-pore
}

\author{
Aniket Bhattacharya,, , $\rightarrow$ William H. Morrison, ${ }^{1}$ Kaifu Luo, ${ }^{2}$ Tapio \\ Ala-Nissila, ${ }^{2,3}$ See-Chen Ying, ${ }^{3}$ Andrey Milchev, ${ }^{4}$ and Kurt Binder ${ }^{5}$ \\ ${ }^{1}$ Department of Physics, University of Central Florida, Orlando, Florida 32816-2385, USA \\ ${ }^{2}$ Department of Applied Physics, Helsinki University of Technology, \\ P.O. Box 1100, FIN-02015 TKK, Espoo, Finland \\ ${ }^{3}$ Department of Physics, Box 1843, Brown University, Providence, Rhode Island 02912-1843, USA \\ ${ }^{4}$ Institute of Physical Chemistry, Bulgarian Academy of Sciences, \\ Georgi Bonchev Street, Block 11, 1113 Sofia, Bulgaria \\ ${ }^{5}$ Institut für Physik, Johannes Gutenberg Universität Mainz, Staudinger Weg 7, 55099, Mainz, Germany
}

(Dated: August 24, 2021)

\begin{abstract}
We investigate several scaling properties of a translocating homopolymer through a thin pore driven by an external field present inside the pore only using Langevin Dynamics (LD) simulations in three dimensions (3D). Motivated by several recent theoretical and numerical studies that are apparently at odds with each other, we determine the chain length $(N)$ dependence scaling exponents of the average translocation time $\langle\tau\rangle$, the average velocity of the center of mass $\left\langle v_{C M}\right\rangle$, and the effective radius of gyration $\left\langle\tilde{R}_{g}\right\rangle$ during the translocation process defined as $\langle\tau\rangle \sim N^{\alpha},\left\langle v_{C M}\right\rangle \sim N^{-\delta}$, and $\tilde{R}_{g} \sim N^{\bar{\nu}}$ respectively, and the scaling exponent of the translocation coordinate (s-coordinate) as a function of the translocation time $\left\langle s^{2}(\tau)\right\rangle \sim \tau^{\beta}$. We find $\alpha=1.36 \pm 0.01, \beta=1.60 \pm 0.01$ for $\left\langle s^{2}(\tau) \sim \tau^{\beta}\right\rangle$ and $\bar{\beta}=1.44 \pm 0.02$ for $\left\langle\Delta s^{2}(\tau)\right\rangle \sim \tau^{\bar{\beta}}, \delta=0.81 \pm 0.04$, and $\bar{\nu} \simeq \nu=0.59 \pm 0.01$, where $\nu$ is the equilibrium Flory exponent in 3D. Therefore, we find that $\langle\tau\rangle \sim N^{1.36}$ is consistent with the estimate of $\langle\tau\rangle \sim\left\langle R_{g}\right\rangle /\left\langle v_{C M}\right\rangle$. However, as observed previously in MC calculations by Kantor and Kardar (Y. Kantor and M. Kardar, Phys. Rev. E, 69, 021806 (2004)) we also find the exponent $\alpha=1.36 \pm 0.01<1+\nu$. We also observe that $\alpha=1.36$ is in closer agreement with $\alpha \simeq(1+2 \nu) /(1+\nu)$ as recently proposed by Vocks et al. (H. Vocks, D. Panja, G. T. Barkema, and R. C. Ball, J. Phys.: Condens. Matter 20, 095224 (2008)). We also discuss the dependence of the scaling exponents on the pore geometry for the range of $N$ studied here.
\end{abstract}

PACS numbers: 87.15.A-, 87.15.H-, 36.20.-r

\section{INTRODUCTION}

Translocation of biopolymers accros a biomembrane, e.g., transport of RNA molecules out of a nucleus, invasion of viruses into cells, etc., are ubiquitous and important processes in biological systems[1]. Recently voltage driven translocation of a single stranded DNA through a $\alpha$-hemolysin pore in biomembrane [2], and subsequently double stranded DNA through synthetic silicon nanopores [3] have stimulated a lot of activities as the phenomenon is rich in fundamental science involved and its prospective technical applications for detecting DNA/RNA sequences. While it is the attributes of heteropolymer translocation that are the key ingredients for prospective new sequencing methods, these experiments have generated stimulating theoretical and numerical studies directed towards fundamental physics of homopolymer translocation through a nanopore. An important question that has been repeatedly raised is how does the average translocation time scale with the chain length and what is the equation of motion that describes the situation adequately. Approaches using FokkerPlanck equation with entropic barrier term incorporated in the free energy have generated useful insights to the problem [4]-11] More recently partial Fokker-Planck equation (PFPE) has been suggested is the natural lan- guage of the problem 9, 10]. Quite naturally, a number of simulational studies have been directed to test predictions of these theories [7]-[22].

This paper is aimed at determining the relevant scaling exponents of forced translocation of a homopolymer through a nanopore by carrying out large scale Langevin dynamics (LD) simulations in three dimensions (3D) and comparing the findings with those predicted by theoretical arguments. We look at the arguments for the unbiased case first as it serves as the reference for extending the theoretical arguments in the presence of an external field. Naturally, the equilibrium radius of gyration $R_{g} \sim N^{\nu}$ of a chain of length $N$, where $\nu$ is the Flory exponent, is used as the relevant length scale in all the theories. The first theoretical argument came from Chuang et al. [7] who predicted that for the unbiased translocation the mean translocation time should scale in the same manner as a freely diffusing chain so that $\langle\tau\rangle \sim R_{g}^{2} / D \sim N^{1+2 \nu}$, assuming the diffusion coefficient $D \sim 1 / N$ appropriate for the free-draining limit (no hydrodynamic interaction). In this theory it is argued that the Rouse relaxation serves as the lower bound and in presence of a nanopore a smaller amplitude accounts for the slowness of the process 14]. This theory also predicts that the scaling exponent of the reaction coordinate defined as $\left\langle s^{2}(\tau)\right\rangle \sim \tau^{\beta}$ is given by $\beta=2 / \alpha$. As 
usual[4]-11] we denote by $s(\tau)$ the monomer that is inside the pore at time $\tau$. Noticeably, the theory is essentially very simple and the exponents are functions of $\nu$ only with $\alpha=1+2 \nu, \beta=2 /(1+2 \nu)$ so that $\alpha \beta=2$. In two dimensions $(2 D) \nu=0.75$ leads to $\alpha=2.5$ and $\beta=0.8$ respectively. In three dimensions (3D) $\nu=0.588$ leads to $\alpha=2.2$ and $\beta=0.92$ respectively. The theory put forward by Dubbledam et al. invokes an additional surface exponent term $\gamma_{1} 25$ so that for the diffusive case this theory predicts [9, 10] $\alpha=2(1+\nu)-\gamma_{1}$ and $\beta=2 / \alpha$. For unbiased translocation this theory also predicts the product $\alpha \beta=2$. Several recent numerical studies in $2 D$ [7, 15, 16, 17] and one in 3D [18] supports Chuang et al., while Dynamic Monte Carlo (DMC) results by Dubbledam et al. report $\alpha=2.5$ and $\beta=0.8$ in $3 \mathrm{D}$ which contradicts Chuang et al. and supports their own prediction [9]. While all the simulation studies verify $\alpha \beta=2.0$, recent theories by Panja et al. and Vocks et al. pointed out the role of decay time of monomer density near the pore and argues that the translocation time is anomalous up to the Rouse time $t_{R} \sim N^{1+2 \nu}$, and becomes diffusive afterwards 12,13$]$. Therefore, for the unbiased translocation the collective numerical results do not support any of the proposed theories completely.

Let us now look at the theoretical studies of driven translocation whose numerical verification including the underlying assumptions is the main focus of the paper. According to Kantor and Kardar $\langle\tau\rangle \sim\left\langle R_{g}\right\rangle /\left\langle v_{C M}\right\rangle \sim$ $N^{1+\nu}$, assuming $v_{C M} \sim 1 / N$. Kantor and Kardar [8] argued that since the chain is only driven at one point inside the narrow pore, the accompanying change in its shape due to the bias is insignificant for the rest of the chain and therefore, the chain in this case is also described by the equilibrium Flory exponent $\nu$. To verify their scaling argument Kantor and Kardar carried out Lattice MC simulation of self-avoiding chains in $2 D$ and noticed that the numerical exponent $\simeq 1.5<1+\nu=1.75$. They argued that finite size effects are severe in this case and the relation $\langle\tau\rangle \sim N^{1+\nu}$ should be taken as an upper bound that will be seen only for the extremely large chains. Vocks et al. on the contrary, using arguments about memory effects in the monomer dynamics came up with an alternate estimate 13$]\langle\tau\rangle \sim N^{\frac{1+2 \nu}{1+\nu}}$. This seems to be consistent with most of the numerical data in 3D. However this estimate fail to capture the recent $2 \mathrm{D}$ simulation results using Langevin dynamics and MC simulations [16, 17] where one sees a crossover of the $\alpha$-exponent from 1.5 to 1.7 (as opposed to 1.428). Dubbledam et al. have extended their PFPE based theory for the driven translocation [10] and came up with the following relations $\alpha=2 \nu+1-\gamma_{1}$ and $\beta=4 /\left(2(1+\nu)-\gamma_{1}\right)$. The prediction of Dubbledam et al. for the exponents are $\alpha=1.55$ and $\beta=1.56$ in $2 D$ and $\alpha=1.5$, and and $\beta=1.6$ in 3D respectively. The DMC results of Dubbledam et al. are consistent with this theory. However, more recent numerical results using $\mathrm{LD}$ and $\mathrm{MD} 22,23$, produce similar results which are only in partial agreement with these theories.

In this paper not only we calculate these scaling exponents $\alpha$ and $\beta$ for the driven chain but provide insights how the scaling aspects are affected by boundary and geometric factors by monitoring some of the relevant time dependent quantities during the translocation process. This allows us to check how well some of the assumptions are satisfied for the driven translocation and discuss possible scenarios for the disagreements between the theoretical predictions and numerical studies. Thus far these issues have not been adequately addressed in the literature.

\section{THE MODEL}

We have used the "Kremer-Grest" bead spring model to mimic a strand of DNA 24]. Excluded volume interaction between monomers is modeled by a short range repulsive LJ potential

$$
\begin{aligned}
U_{L J}(r) & =4 \varepsilon\left[\left(\frac{\sigma}{r}\right)^{12}-\left(\frac{\sigma}{r}\right)^{6}\right]+\varepsilon \text { for } r \leq 2^{1 / 6} \sigma \\
& =0 \text { for } r>2^{1 / 6} \sigma .
\end{aligned}
$$

Here, $\sigma$ is the diameter of a monomer, and $\varepsilon$ is the depth of the potential. The connectivity between neighboring monomers is modeled as a Finite Extension Nonlinear Elastic (FENE) spring with

$$
U_{F E N E}(r)=-\frac{1}{2} k R_{0}^{2} \ln \left(1-r^{2} / R_{0}^{2}\right),
$$

where $r$ is the distance between consecutive monomers, $k$ is the spring constant and $R_{0}$ is the maximum allowed separation between connected monomers. We use the Langevin dynamics with the equation of motion

$$
\ddot{\vec{r}}=-\vec{\nabla} U_{i}-\Gamma \dot{\vec{r}}_{i}+\vec{W}_{i}(t) .
$$

Here $\Gamma$ is the monomer friction coefficient and $\vec{W}_{i}(t)$, is a Gaussian white noise with zero mean at a temperature $\mathrm{T}$, and satisfies the fluctuation-dissipation relation:

$$
<\vec{W}_{i}(t) \cdot \vec{W}_{j}\left(t^{\prime}\right)>=6 k_{B} T \Gamma \delta_{i j} \delta\left(t-t^{\prime}\right) .
$$

The purely repulsive wall consists of one monolayer of LJ particles of diameter $1.5 \sigma$ on a triangular lattice at the $x y$ plane at $z=0$. The pore is created by removing the particle at the center. Inside the pore, the polymer beads experience a constant force $F$ and a repulsive potential from the inside wall of the pore. The reduced units of length, time and temperature are chosen to be $\sigma, \sigma \sqrt{\frac{m}{\varepsilon}}$, and $\varepsilon / k_{B}$ respectively. For the spring potential we have chosen $k=30$ and $R_{i j}=1.5 \sigma$, the friction coefficient $\Gamma=1.0$, and the temperature is kept at $1.5 / k_{B}$ throughout the simulation. 


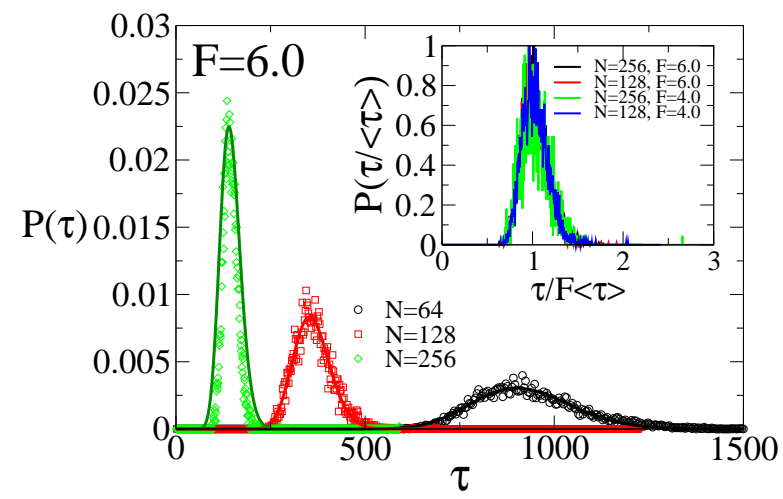

FIG. 1: Histogram $P(\tau)$ of flight times for chain lengths $N=64,128$, and 256 for bias $\mathrm{F}=6.0$. The symbols represent simulation data and the solid lines are fits with a form $P(\tau)=A \tau^{\lambda} \exp (-\mu \tau)$. The inset shows the corresponding scaled plots where the $\tau$-axis has been scaled by $F\langle\tau\rangle$ and the $y$-axis has been scaled by the maximum value of the histogram.

We carried out simulations for chain lengths $N$ from $8-256$ for two choices of the biasing force $F=4$ and 6 , respectively. Initially the first monomer of the chain is placed at the entry of the pore. Keeping the first monomer in its original position the rest of the chain is then equilibrated for times at least an amount proportional to the $N^{1+2 \nu}$. The chain is then allowed to move through the pore driven by the field present inside the pore. When the last monomer exits the pore we stop the simulation and note the translocation time and then repeat the same for 5000 such trials.

\section{SIMULATION RESULTS AND THEIR INTERPRETATION}

Typical histograms for the passage time are shown in Fig. 1 for $F=6.0$. When the time axis is scaled by the mean translocation time multiplied by the bias $F$ and the peak of the distribution is normalized to unity, we observe (inset) a nice scaling of all the histograms on a single master curve. We also note that an excellent fit (solid lines) could be made with an expression $P(\tau)=A \tau^{\lambda} \exp (-\mu \tau)$ for all the plots with the peak position being given by $\tau_{\max }=\lambda / \mu$. We calculated the average translocation time from the weighted mean $\langle\tau\rangle=\int_{0}^{t_{\max }} \tau P(\tau) d \tau$, where $t_{\max }$ for each distribution is chosen such that at $t_{\max }$ the distribution $P(\tau)$ is about $0.01 \%$ of its peak value. We have checked that $\langle\tau\rangle$ calculated from the area is marginally greater than $\tau_{\text {peak }}$ obtained from $P(\tau)$.

The scaling exponent $\alpha$ of the mean translocation time $\langle\tau\rangle \sim N^{\alpha}$ is extracted by plotting the $\langle\tau\rangle$ as a function of $N$ shown in Fig. 2. Evidently, we find that $\langle\tau\rangle \sim 1 / F$ and $\langle\tau\rangle \sim N^{1.36}$. The inset of Fig. 2 shows

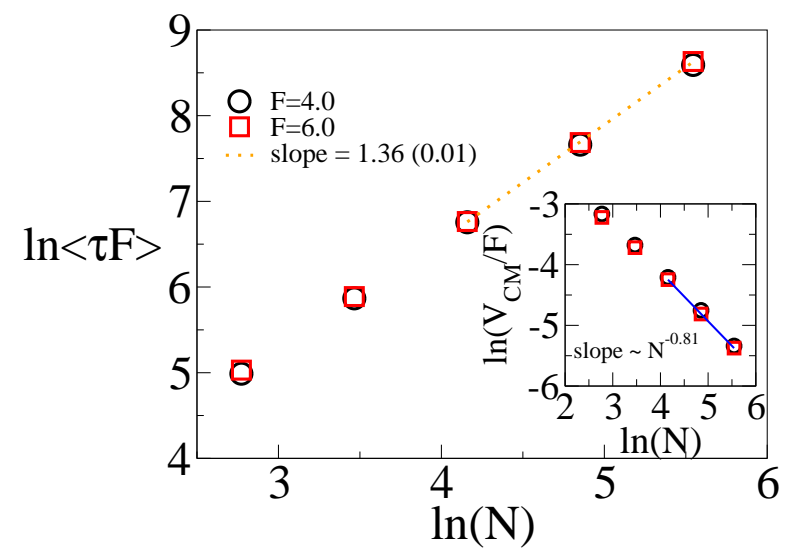

FIG. 2: Scaling of the mean translocation time $\langle\tau\rangle$ (logarithmic scale) scaled by the applied bias $F$ as a function of chain length $N$ (logarithmic scale). The open circles and squares refer to $F=4.0$ and $F=6.0$ respectively. The inset shows the corresponding scaling of $v_{C M} / F$.

that the velocity of the center of mass increases linearly with the bias and scales as $v_{C M} \sim 1 / N^{0.81}$. We note that $v_{C M}$ does not scale as $1 / N$. It has been suggested that this exponent is not universal and depends on the width and the geometry of the pore 22]. We will come back to this issue later. The scaling exponent $\beta$ of the $s$ coordinate is shown in Fig. 3. For clarity, we have shown results for the two largest chain lengths $N$ $=128$ and 256. When we calculate the first and the second moments of $s(\tau)$ we find that $\langle s(\tau)\rangle \sim \tau^{0.8}$ and $\left\langle s^{2}(\tau)\right\rangle \sim \tau^{1.6}$ for a wide range of the translocation time (the slope remains the same between the blue and the green vertical windows and between the green and the red vertical windows respectively in Fig. 3). The data as a function of the scaled translocation time $F \tau$ show excellent collapse. Since $\left\langle s^{2}(\tau)\right\rangle \sim(\langle s(\tau)\rangle)^{2}$, one expects to see $\left\langle\Delta s^{2}(\tau)\right\rangle=\left\langle s^{2}(\tau)-\langle s(\tau)\rangle^{2}\right\rangle \sim \tau^{1.6}$ during the same time window. However, $\left\langle s^{2}(\tau)-\langle s(\tau)\rangle^{2}\right\rangle$ reveals additional features where the slope changes from $\left\langle s^{2}(\tau)-\langle s(\tau)\rangle^{2}\right\rangle \sim \tau^{1.03}$ (between blue and green dashed vertical lines) to $\left\langle s^{2}(\tau)-\langle s(\tau)\rangle^{2}\right\rangle \sim \tau^{1.44}$ (between green and red vertical lines). For the forced translocation $\langle s(\tau)\rangle \neq 0$ and it is likely that a tiny difference of time dependence of the first and 2nd moment during the translocation of the chain that is not visible in the plot of 1 st or the 2 nd moment of the $s$-coordinate becomes noticeable in its fluctuation. Therefore, if we use the fluctuations in $s$ to define $\left\langle(s(\tau)-\langle s(\tau)\rangle)^{2}\right\rangle \sim \tau^{\bar{\beta}}$, then from the late time slope (Fig. 3) then we get $\bar{\beta}=1.44$.

We now compare these results with the theoretical predictions and other existing numerical results. The translocation exponent in 3D, according to Kantor and Kardar [8], is $1+\nu=1.588$, and according to Dubbledam 10] is 1.50. First of all, as observed in $2 D$ MC simulations by Kantor and Kardar [8] we also obtain 


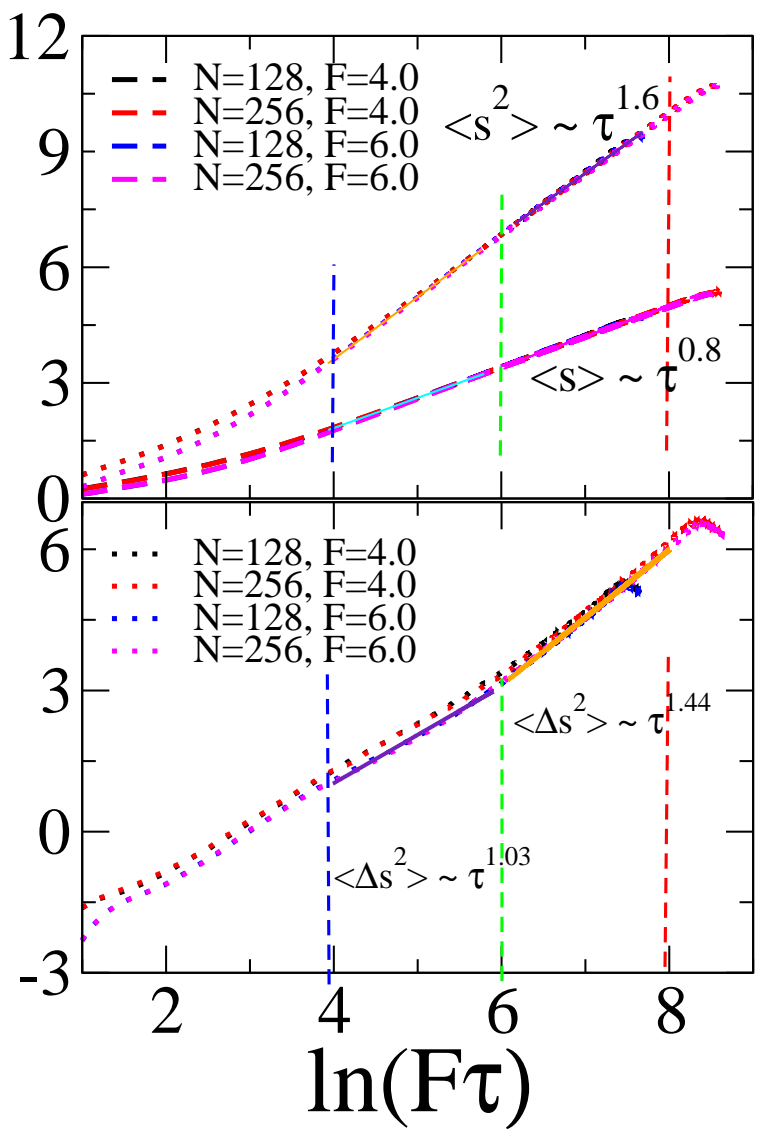

FIG. 3: variation of $\left\langle s^{2}(\tau)\right\rangle$ (top, dotted) and $\langle s(\tau)\rangle$ (top, dashed-dot), and $\left\langle s^{2}(\tau)-\langle s\rangle^{2}\right\rangle$ (bottom) as a function of the scaled translocation time $F \tau$. The black and blue colors correspond to chain length $\mathrm{N}=128$ for $\mathrm{F}=4.0$ and $\mathrm{F}=6.0$ respectively. The red and magenta colors correspond to the chain length $\mathrm{N}=256$ for $\mathrm{F}=4.0$ and $\mathrm{F}=6.0$ respectively.

a smaller value of $\alpha=1.36 \pm 0.01<1+\nu=1.588$ in 3D. Kantor and Kardar argued that a lower value is obtained due to finite size effects and expect that for very large chains one would find $1+\nu \simeq 1.59$. This bound has recently been criticized by Vocks et al. 113] who using arguments about memory effects in the monomer dynamics came up with an alternate exponent estimate given by $\frac{1+2 \nu}{1+\nu}=1.37$. Evidently, our result is in agreement with this prediction. As for the exponent $\beta$ we find $\left\langle s^{2}(\tau)\right\rangle \sim \tau^{1.6}$, and $\left\langle\Delta s^{2}(\tau)\right\rangle=\left\langle s^{2}(\tau)-\langle s\rangle^{2}\right\rangle \sim \tau^{1.44}$ (if we use the later window). Therefore, with Dubbledam et al. we do not agree with the calculated value of $\alpha$, but Dubbledam et al. also used $\left\langle s^{2}(\tau) \sim \tau^{\beta}\right.$, to define the exponent $\beta$ and the numerical value $\beta=1.6$ is exactly the same as found here. It is noteworthy that the fluctuation $\left\langle\Delta s^{2}(\tau)\right\rangle$ is time dependent and the slope of $\left\langle\Delta s^{2}(\tau)\right\rangle \sim \tau^{1.03}$ at early time crosses over to $\left\langle\Delta s^{2}(\tau)\right\rangle \sim t^{1.44}$ at a later time, while the slope for $\left\langle s^{2}(\tau)\right\rangle \sim \tau^{1.6}$ is constant for a wider range. If we use $\beta=1.44$, obtained from the definition of fluctuation of the $s$ coordinate, then we find the relation $\alpha \beta=2.0$ is satisfied for the forced translocation as well. This trend is qualitatively the same for the simulation using a square pore [23], where we find that $\langle\tau\rangle \sim N^{1.41},\left\langle s^{2}(\tau)\right\rangle \sim \tau^{1.52}$, and $\left\langle\Delta s^{2}(\tau)\right\rangle \sim \tau^{1.45}$ (so that $\alpha \beta \simeq 2.0$, same as reported here if we extract $\beta$ from the slope of the plot $\left.\left\langle\Delta s^{2}(\tau)\right\rangle \sim \tau\right)$. Our results may be relevant in the context of a recent recent article by Chatelain, Kantor, and Kardar 27] who showed that the variance of the probability distribution $P(s, t)$ grows subdiffusievly.

We now look more closely at the factors responsible for the translocation process. The expression $\tau \sim$ $\left\langle R_{g}\right\rangle /\left\langle v_{C M}\right\rangle \sim N^{1+\nu}$ has two components: the dependence of $v_{C M}$ on $N$ and $R_{g}$ on $N$ respectively. We now look at these two components separately. During the driven translocation the chain does not find enough time to relax. Therefore, it is important to know how does the shape of the chain vary as a function of time and how different it is compared to its equilibrium configuration. During the forced translocation at any instant of time only one segment of the entire chain feels the bias. Kantor and Kardar 8] argued that the shape of the chain is hardly affected by it so that it will still be described by the equilibrium Flory exponent $\nu$. This argument will not be strictly valid for the model used here as the beads are connected by elastic bonds and it is expected that quite a few neighbors on either side of the driven bead inside the pore will be indirectly affected by it.

In order to verify this issue first, we have calculated the equilibrium $\left\langle R_{g}\right\rangle$ of the chain clamped at one end at the pore in presence of the same LJ wall. We find $\nu \sim 0.6 \pm 0.01$ (Fig. 44). We have also calculated the relaxation time $\tau_{r}$ of the end-to-end vector $\left\langle\left(\mathbf{R}_{1 \mathrm{~N}}(\mathrm{t}+\tau)-\langle\mathbf{R}\rangle\right) \cdot\left(\mathbf{R}_{1 \mathrm{~N}}(\mathrm{t}+\tau)-\langle\mathbf{R}\rangle\right)\right\rangle \sim \exp \left(-\mathrm{t} / \tau_{\mathrm{r}}\right)$ and checked that we get the same $\nu$ from the relaxation measurements. This is consistent with the theoretical prediction of Eisenriegler, Kremer, and Binder that in presence of the wall the exponent $\nu$ remains the same as that of its bulk counterpart [26]. To get an idea how fast is the translocation process, compared to the corresponding relaxation time, for the chain lengths $N=64,128$, and 256, we find $\tau_{r} \sim 1000,4500$, and 20200 respectively and the corresponding average translocation times $\langle\tau\rangle$ are 215,530 , and 1330 , respectively. Even in the linear response regime where $\langle\tau\rangle \sim 1 / F$, we observe $\tau_{r}>>\langle\tau\rangle$. The insets of Fig. 4 shows the time dependence of $\tilde{R}_{g}(t)$ (we use a different notation $\tilde{R}_{g}$ for the driven chain). We notice that during the translocation process the chain is significantly elongated around $t \simeq 0.5\langle\tau\rangle$ and acquires relatively compact structure immediately upon exiting the pore. The dashed lines (black and green) show the corresponding average values $\tilde{R}_{g}$ from which we extract the exponent $\bar{\nu} \simeq \nu$ (Fig. (4). Contrary to what is assumed by Kantor and Kardar, we notice significant distortion of the chain. Surprisingly, we find that the $\tilde{R}_{g}$ scales almost the same way as $\left\langle R_{g}\right\rangle \sim N^{0.6}$ (slopes are 


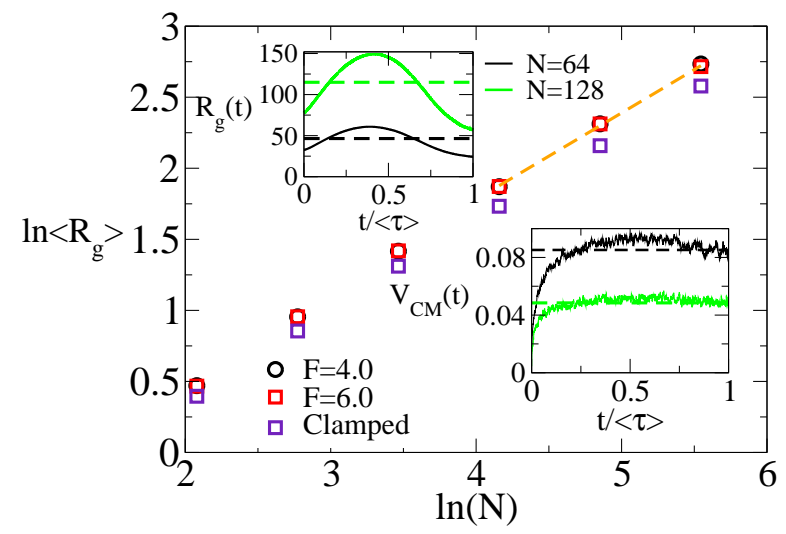

FIG. 4: Equilibrium $R_{g}$ and effective $\tilde{R}_{g}$ during the translocation process. The absolute value of the effective $\tilde{R}_{g}$ is larger than the equilibrium value as it is pulled, but both of them scale with the equilibrium Flory exponent. The insets shows the average time dependence of the $v_{C M}(t)$ and $R_{g}(t)$ during the translocation. The straight lines in the inset represent the average value.

the same in Fig. 4). even when $\langle\tau\rangle<<\tau_{r}$. Therefore, numerically we find that the chain is still described by the equilibrium $\left\langle R_{g}\right\rangle$.

Likewise, as expected in LD simulation, we notice that $v_{C M}(t)$ saturates quite quickly and this value is almost the same during the translocation process and $\simeq\left\langle v_{C M}\right\rangle \sim 1 / N^{\delta}$. where $\delta=0.81 \pm 0.04$. Since $\bar{\nu} \simeq \nu$, our studies indicate that it is the $\left\langle v_{C M}\right\rangle$ which does not exhibit inverse linear dependence on chain length $N$ is the responsible factor for the deviation from $\langle\tau\rangle \sim N^{1+\nu}$.

It is worth mentioning that we have carried out exactly the same LD simulations with wall particles on a square lattice 23]. We find that $\left\langle\tau \sim N^{1.41},\left\langle s^{2}(\tau)\right\rangle \sim \tau^{1.52}\right.$, and $\left\langle\Delta s^{2}(\tau)\right\rangle \sim \tau^{1.45}$ (so that $\alpha \beta \simeq 2.0$, same as reported here if we extract $\beta$ from the slope of the plot $\left.\left\langle\Delta s^{2}(\tau)\right\rangle \sim \tau\right)$. These results for the square pore are also consistent with recently reported LD and MD simulation results in 3D using GROMACS 22]. Recently Gauthier et al. 21] carried out similar studies of polymer translocation through a narrow pore (including hydrodynamic interactions) and found a systematic variation of the measured scaling exponents as a function of the pore width. However, their studies are limited to relatively narrow range of $N$ up to 31 only. In our studies the exponents for a relatively wide range of $N$ seem to depend on the pore geometry. Whether this implies true nonuniversality or not remains an open issue.

\section{CONCLUSION}

To summarize, we have used Langevin dynamics in 3D to study the scaling properties of a driven translocating chain through a nanopore. We notice that the chain un- dergoes a significant shape change during the fast translocation process, contrary to what assumed by Kantor and Kardar is formulating the theory of forced translocation. However, despite significant distortion, we find the chain is still described by the equilibrium Flory exponent. We find that the $\left\langle v_{C M}\right\rangle$ does not scale as its bulk counterpart and depends on pore width and geometry. It is likely that density variation on either side of the pore during the translocation process affects the overall motion of the chain. We find that the $\alpha=1.36<1+\nu$. It is worth mentioning that the collective numerical work by various groups failed to validate the Kantor and Kardar result $\alpha=1+\nu$ for the forced translocation, including the results listed here. Likewise, although the value of $\alpha=1.36$ that we obtain is in excellent agreement with the analytical estimate of Vocks et al. $\alpha=\frac{1+2 \nu}{1+\nu}=1.37$ in $3 \mathrm{D}$, the results from $2 \mathrm{D}$ simulations do not agree with the estimate of Vocks et al. . Finally, we notice a difference in the $s$-exponent $\beta$ when calculated from its second moment $(\beta=1.6)$ and its fluctuations $(\bar{\beta}=1.44)$. The later $(\bar{\beta}=1.44)$ agrees with $\alpha \bar{\beta} \simeq 2.0$ while $\beta=1.6$ overestimates it $(\alpha \beta \simeq 2.2>2.0)$. The fluctuations $\left\langle\Delta s^{2}(\tau)\right\rangle$ seem to reveal more structures not adequately studied so far. When we compare these results with the existing theories and other numerical results we notice that these results only partially support one theory or the other. Certainly more numerical and analytic work are needed for a more comprehensive understanding of forced translocation through nanopore.

\section{ACKNOWLEDGEMENT}

A. B. gratefully acknowledges the local hospitality of the Institut für Physik, Johannes-Gutenberg Universität, Mainz, the travel support from the Deutsche Forschungsgemeinschaft, SFB 625/A3, and the local hospitality and travel support from the COMP Center of Excellence, Helsinki University of Technology respectively, and thanks Prof. M. Muthukumar for valuable discussions. T.A-N. and K.L. have been in part supported by the Academy of Finland through the COMP Center of Excellence program and TransPoly consortium grant.

* Author to whom the correspondence should be addressed; Electronic address: aniket@physics.ucf.edu

[1] B. Alberts et al., Molecular Biology of the Cell (Garland Publishing, New York, 1994).

[2] J. J. Kasianowitch, E. Brandin, D. Branton, and D. Deamer, Proc. Natl. Acad. Sci. U.S.A. 93, 13770 (1996); A. Meller, L. Nivon, E. Brandin, J. Golovchenko, and D. Branton, ibid 97, 1097 (2000);

[3] J. L. Li, M. Gershow, D. Stein, E. Brandin, and J. A. Golovchenko, Nat. Mater. 2, 611 (2003); A. J. Storm, J. 
H. Chen, X. S. Ling, H. W. Zandbergen, and C. Dekker, ibid 2, 537 (2003).

[4] W. Sung and P. J. Park, Phys. Rev. Lett. 77, 783 (1996)

[5] M. Muthukumar, J. Chem. Phys. 111, 10371 (1999).

[6] D. K. Lubensky and D. Nelson, Biophys. J. 77, 1824 (1999)

[7] J. Chuang, Y. Kantor, and M. Kardar, Phys. Rev. E, 65, 011802 (2001).

[8] Y. Kantor and M. Kardar, Phys. Rev. E, 69, 021806 (2004).

[9] J. L. A. Dubbledam, A. Milchev, V. G. Rostiashvili, and T. Vilgis, Phys. Rev. E 76, 010801(R) (2007)

[10] J. L. A. Dubbledam, A. Milchev, V. G. Rostiashvili, and T. Vilgis, Europhysics Letters 7918002 (2007).

[11] J. K. Wolterink, G. T. Barkema, and D. Panja, Phys. Rev. Lett. 96, 208301 (2006).

[12] D. Panja, G. T. Barkema, and R. C. Ball, J. Phys.: Condens. Matter 19, 432202 (2007); ibid20, 075101 (2008).

[13] H. Vocks, D. Panja, G. T. Barkema, and R. C. Ball, J. Phys.: Condens. Matter 20, 095224 (2008).

[14] A. Milchev, K. Binder, and A. Bhattacharya, J. Chem. Phys. 121, 6042 (2004).

[15] K. Luo, T. Ala-Nissila, and S-C. Ying, J. Chem. Phys. 124, 034714 (2006).

[16] K. Luo, T. Ala-Nissila, and S-C. Ying, J. Chem. Phys. 124, 114704 (2006).
[17] I. Huopaniemi, K. Luo, T. Ala-Nissila, S-C. Ying, J. Chem. Phys. 125, 124901 (2006).

[18] D. Wei, W. Yang, X. Jin, and Q. Liao, J. Chem. Phys. 126, 204901 (2007)

[19] K. Luo, T. Ala-Nissila, and S-C. Ying, and Aniket Bhattacharya J. Chem. Phys. 126145101 (2006); Phys. Rev. Lett. 99148102 (2007); ibid 100058101 (2008).

[20] S. Matysiak, A. Montesi, M. Pasquali, A. . Kolomeisky, C. Clementi, Phys. Rev. Lett. 96118103 (2006).

[21] M. G. Gauthier and G. W. Slater, Eur. Phys. J. E 25, 17 (2008).

[22] K. Luo, et al. arXiv;cond-mat/0805.4312 (to appear in Phys. Rev. E)

[23] Aniket Bhattacharya (unpublished). Here the wall consists of a monolayer of LJ particles of $\sigma=1$ arranged on a square lattice and the pore is created by removing 4 particles around the center.

[24] G. S. Grest \& K. Kremer, Phys. Rev. A 33, 3628 (1986);

[25] R. Hegger and P. Grassberger, J. Phys. A 27, 4069 (1994); M. N. Barber, ibid 11, 1833 (1978).

[26] E. Eisenriegler, K. Kremer, and K. Binder, J. Chem. Phys. 77, 6296 (1982); P. G. de Gennes, Macromol 13, 1069 (1980).

[27] C. Chatelain, Y. Kantor, and M. Kardar, arXiv;condmat/0805.4168v1, Phys. Rev. E 78, 021129, 2008. 